TAKUMASA KONDO $(*)\left({ }^{\circ}\right)$ - ANDREA AMALIA RAMOS-PORTILLA $(* *)$

ANA LUCIA B.G. PERONTI $(* * *)$ - PENNY J. GULLAN $(* * * *)$

\title{
KNOWN DISTRIBUTION AND PEST STATUS OF FLUTED SCALE INSECTS (HEMIPTERA MONOPHLEBIDAE ICERYINI) IN SOUTH AMERICA $\left({ }^{1}\right)$
}

\author{
(*) Laboratorio de Entomología, Corporación Colombiana de Investigación Agropecuaria (CORPOICA), Centro de \\ Investigación Palmira, Valle del Cauca, Colombia. \\ (**) Instituto Colombiano Agropecuario ICA, Museo Entomológico UNAB-Universidad Nacional de Colombia, sede \\ Bogotá. \\ (***) Universidade Estadual Paulista Júlio de Mesquita Filho, Faculdade de Ciências Agrárias e Veterinárias de \\ Jaboticabal. \\ (****) Division of Evolution, Ecology \& Genetics, Research School of Biology, The Australian National University, \\ Canberra, A.C.T. 2601, Australia. E-mail: penelope.gullan@anu.edu.au \\ $\left.{ }^{\circ}\right)$ Corresponding author: E-mail: takumasa.kondo@gmail.com
}

Kondo T., Ramos-Portilla A.A., Peronti A.L.B.G., Gullan P.J. - Known distribution and pest status of fluted scale insects (Hemiptera Monophlebidae Iceryini) in South America.

Current information on the known distribution of all 18 species (12 species of Crypticerya Cockerell and 6 species of Icerya Signoret) of fluted scale insects (Hemiptera: Monophlebidae: Iceryini) found in South America is provided. A list of known host plants and natural enemies of these species is given and their pest status in South America is discussed.

Key Words: Crypticerya, Icerya, Monophlebidae, South America, distribution, host plants.

\section{INTRODUCTION}

The tribe Iceryini (Hemiptera: Monophlebidae) is composed by 84 species distributed in five genera, i.e., Crypticerya Cockerell, Icerya Signoret, Echinicerya Morrison, Gigantococcus Pesson \& Bielenin, and Gueriniella Targioni Tozzetti (GARCía Morales et al., 2016; UnRUH \& GULLAN, 2008a, b). Of these, only Crypticerya and Icerya are known from South America. Twelve of 27 described species of Crypticerya and six of 35 species of Icerya have been recorded from one or more South American countries (GARCíA Morales et al., 2016). For the purpose of this paper, we treat South America as including Argentina, Bolivia, Brazil, Chile, Colombia, Ecuador, French Guiana, Guyana, Paraguay, Peru, Suriname, Uruguay, and Venezuela, but exclude the ABC islands, Trinidad and Tobago, and Panama, which are sometimes considered as part of South America. In South America, the pest iceryine species are commonly called fluted scales because the female (often hermaphroditic) produces a white waxy ovisac marked with longitudinal grooves or flutes. The species of greatest economic importance in South America are Crypticerya brasiliensis (Hempel), C. multicicatrices Kondo \& Unruh, C. genistae (Hempel), C. montserratensis (Riley \& Howard), C. palmeri (Riley \& Howard), C. zeteki (Cockerell), Icerya purchasi Maskell and I. seychellarum (Westwood). All of the above species are polyphagous and attack plant species of economic importance. For example, C. multicicatrices is known to feed on almost 150 plant

\footnotetext{
${ }^{1}$ Original scientific contribution presented and discussed at XIV International Symposium on Scale Insect Studies, Catania-Italy, 13-16 June 2016.
}

species and, when outbreaks occurred on San Andres Island, Colombia, the most common hosts included essentially all palm species (Arecaceae), breadfruit (Artocarpus altilis (Parkinson) Fosberg), Citrus spp., guava (Psidium spp.), all leguminous trees and weeds (Fabaceae), Ficus spp. (Moraceae), Mammea americana L. (Calophyllaceae), Melicoccus bijugatus Jacq. (Sapindaceae) and Spondias spp. (Anacardiaceae) (Kondo et al., 2012a). Another species, $C$. genistae, has been reported on 80 species of plants, and was reported recently in Colombia, where it is considered a pest of chili peppers (Kondo et al., 2016). We provide information on the distribution and host plants of the Iceryini species found in South America and discuss their economic importance.

\section{MATERIAL AND METHODS}

The present paper summarizes our current understanding of the distribution, host plants, pest status, natural enemies, and other information such as biological and taxonomic notes on scale insects of the tribe Iceryini (Hemiptera: Monophlebidae) in South America based on a literature review and personal observations.

\section{RESULTS AND DISCUSSION}

LIST OF ICERYINE SPECIES KNOWN FROM SOUTH AMERICA

\section{Crypticerya abrahami (Newstead)}

Llaveia abrahami Newstead, 1917: 1.

Distribution In South AMERICA - Colombia (Kondo \& Unruh, 2009), Guyana (Foldi, 2009; Newstead, 1917; UNRUH \& GULLAN, 2008b), Venezuela (FoLdI, 2009). 
Pest STATUS - Not considered a pest.

NATURAL EnEmies - Unknown.

Host Plants - Euphorbiaceae: Sapium jenmanii Hemsl. (Unruh \& Gullan, 2008b); Fabaceae: Pithecellobium dulce (Roxb.) Benth. (NewsteAd, 1917); Myrtaceae: Psidium guajava L. (T. Kondo, personal observation).

REMARKS - The insects live underneath the bark of their host plant; adult females are covered by a thin layer of white wax and lack the typical ornamental waxy secretions seen in many species of Crypticerya. Instead of an ovisac, the females keep their eggs underneath their body in a marsupium, which may be an adaptation for living in crowded spaces. According to the original description by Newstead (1917), the type specimens were found "inhabiting indentations in the bark of a rubber-producing tree, Sapium jenmani Hemsl. (Euphorbiaceae); attended by ants which construct coverings over the Coccids". Similarly, in Colombia they are often found on Pithecellobium dulce and Psidium guajava, underneath the bark, on bark crevices and inside ant cartons and tended by Azteca and Crematogaster (Formicidae) ants (T. Kondo, personal observation). Two other species, $C$. pimentae from Jamaica and $C$. rosae known from Guadeloupe, Haiti, Mexico, Puerto Rico and the U.S.A., are morphologically identical to $C$. abrahami, but are not genetically identical (Unruh \& Gullan, 2008b).

Crypticerya brasiliensis (Hempel)

Icerya brasiliensis Hempel, 1900: 370.

Distribution in South America - Argentina (Lizer y Trelles, 1938), Colombia (Kondo et al., 2016), Brazil (Hempel, 1900, 1912; Lizer y Trelles, 1938; PAlomar et al., 2015; Peronti \& Rosa, 2006; UnRUh \& Gullan, 2008b), Guyana (UNRUH \& GULLAN, 2008a).

PEST STATUS - This species is often found congregating in large numbers on the underneath of branches and twigs of its host, and has been reported as causing the dieback of numerous shade trees in São Paulo, Brazil (HEMPEL, 1900). Recently, in the Department of Boyacá in Colombia, $C$. brasiliensis was reported as causing great damage to many types of shrubs and guava trees (KONDO et al., 2016).

NATURAL Enemies - Hymenoptera: Encyrtidae: Brethesiella abnormicornis (Girault), Brethesiella longipes Blanchard (NOYES, 2004; TRJAPITZIN \& TRJAPITSYN, 2006).

Host Plants - Arecaceae: Caryota sp.; Euphorbiaceae: Codiaeum sp.; Lauraceae: Cinnamomum camphora (L.) J. Presl.; Magnoliaceae: Liriodendron tulipifera L.; Moraceae: Ficus sp.; Myrtaceae: Callistemon viminalis (Sol. ex Gaertn.) G. Don, Psidium guajava L.; Rosaceae: Rosa sp. (Hempel, 1900, 1912; Kondo et al., 2016; Lizer Y Trelles, 1938; Palomar et al., 2015; Peronti \& Rosa, 2006).

ReMARKs - Outside South America, C. brasiliensis has been recorded from Panama (Canal Zone) by CocKerelL (1914). In the State of São Paulo, C. brasiliensis is commonly found on guava and various ornamental plants (PAlomar et al., 2015; Peronti \& Rosa, 2006). In life, the arrangement of waxy secretions in $C$. brasiliensis, $C$. multicicatrices and $C$. zeteki is superficially similar, with all having a long caudal tuft and a shorter cephalic tuft (KONDO et al., 2016). In C. brasiliensis, the waxy tufts laterad to the long caudal tuft are usually three or more times longer than other marginal tufts. However, in C. zeteki, the waxy tufts laterad to the long caudal tuft are about three times longer than the shorter marginal tufts, thus overlapping with $C$. brasiliensis (KoNDO et al., 2016). At this time, the only reliable way to differentiate them is by looking at slidemounted specimens.

\section{Crypticerya flava (Hempel) \\ Icerya flava Hempel, 1920: 334.}

Distribution in South America - Brazil (Hempel, 1920).

Pest Status - Unknown.

NATURAL ENEMIES - Not reported.

Host Plants - Hempel (1920) lists C. flava (as Icerya flava) as occurring on the bark of indigenous trees locally known as 'cambará preta' and 'sucará'. UNRUH \& GULLAN (2008b) identified 'cambará preta' as "black Gochnatia”, a plant in the family Asteraceae. The other plant, 'sucara' appears to be a plant in the genus Xylosma, in the family Salicaceae.

REMARKS - This species is known only from Brazil and very little information is available for it in the scientific literature.

Crypticerya flocculosa (Hempel)

Icerya flocculosa Hempel, 1932: 312.

Distribution in South America - Brazil (Hempel, 1932).

Pest Status - Unknown

Natural EnEmies - Not reported.

Host Plants - Fagaceae: Castanea sp.; Rutaceae: Citrus sp. (HEMPEL, 1932).

REMARKS - Only known from Brazil. UNRUH \& GULLAN (2008b) were unable to examine specimens of $C$. flocculosa for their identification guide to species of Iceryini and suggested that this species may be a synonym of another species.

\section{Crypticerya genistae (Hempel)}

Icerya genistae Hempel, 1912: 55.

Distribution in South America - Brazil (Culik et al., 2007; HeMPEL, 1912), Colombia (Kondo et al., 2016).

PeSt STATUS - Crypticerya genistae has been reported as a pest of eggplants, peanuts, peppers and tomatoes in Barbados (Hodges et al., 2008). In Puerto Rico it has been reported as a devastating pest of soybeans and other legumes (JENKINS et al., 2014). In Florida, hosts currently are restricted to common roadside plants, with no infestations observed in agricultural crops or on ornamental plants (Hodges et al., 2008). In Colombia, it has been reported as a pest of chili peppers, Capsicum sp. cv. 'Topito' (Solanaceae) (Kondo et al., 2016). Plants in the families Asteraceae, Euphorbiaceae and Fabaceae are favored by $C$. genistae (ETIENNE \& MATILE-FerRero 2008; Hodges et al., 2008; STOCKS, 2013) and plants of the families Malvaceae and Solanaceae also appear to be common hosts of $C$. genistae.

Natural Enemies - Coleoptera: Coccinellidae: Anovia circumclusa (Gorham) (Ciomperlik 2010), A. punica Gordon (T. Kondo, personal observation), Rodolia cardinalis (Mulsant) (ETIENNE \& MATILE-FERRERO, 2008). Diptera: Cecidomyiidae: Pectinodiplosis erratica (Felt) (GAgné \& Etienne, 2009), Phoridae: Syneura cocciphila (Coquillet) (CIOMPERLIK, 2010).

Host Plants - Arecaceae: Dypsis lutescens (H. Wendl.) Beentje \& J. Dransf.; Asteraceae: Ambrosia artemisiifolia L., Baccharis halimifolia L., Bidens pilosa L., Centratherum punctatum Cass., Conyza canadensis (L.) Cronquist, Eclipta prostrata (L.) L., Eleutheranthera ruderalis (Sw.) Sch. Bip., Emilia fosbergii Nicolson, Erigeron canadensis L., 
Eupatorium capillifolium (Lam.) Small ex Porter \& Britton, Helianthus debilis Nutt., Heterotheca subaxillaris (Lam.) Britton \& Rusby, Mikania micrantha Kunth, Parthenium hysterophorus L., Pluchea carolinensis (Jacq.) G. Don, Pluchea odorata (L.) Cass., Solidago leavenworthii Torr. \& A. Gray, Sonchus oleraceus L., Sphagneticola trilobata (L.) Pruski, Tridax procumbens L.; Cannabaceae: Trema micrantha (L.) Blume.; Casuarinaceae: Casuarina equisetifolia L.; Convolvulaceae: Ipomoea batatas (L.) Lam., I. pes-caprae (L.) R. Br., Merremia aegyptia (L.) Urb.; Cucurbitaceae: Momordica charantia L.; Cyperaceae: Carex sp., Rhynchospora sp.; Euphorbiaceae: Euphorbia cyathophora Murray, E. heterophylla L., E. hirta L., E. hypericifolia L., E. mesembryanthemifolia Jacq., Ricinus communis L.; Fabaceae: Acacia cornigera (L.) Willd., A. macracantha Humb. \& Bonpl. ex Willd., Aeschynomene americana L., Arachis pintoi Krapov. \& W.C. Greg., Caesalpinia bonduc (L.) Roxb., Cajanus cajan (L.) Huth, Calopogonium mucunoides Desv., Canavalia rosea (Sw.) DC., Centrosema pubescens Benth., C. virginianum (L.) Benth., Crotalaria incana L., C. retusa L., Desmanthus virgatus (L.) Willd., Desmodium incanum (Sw.) DC., D. tortuosum (Sw.) DC., Desmodium sp., Genista scoparia Lam., Haematoxylum campechianum L., Indigofera suffruticosa Mill., I. tinctoria L., Kummerowia striata (Thunb.) Schindl., Macroptilium lathyroides (L.) Urb., Mimosa pigra L., M. pudica L., Pueraria phaseoloides (Roxb.) Benth., Rhynchosia baukea Du Puy \& Labat, Senna obtusifolia (L.) H.S. Irwin \& Barneby, S. polyphylla (Jacq.) H.S. Irwin \& Barneby, Sesbania sp., Stylosanthes biflora (L.) Britton, Sterns \& Poggenb., Teramnus labialis (L. f.) Spreng., Vicia acutifolia Elliott, Vigna luteola (Jacq.) Benth., Vigna sp.; Malvaceae: Malvastrum sp., Waltheria indica L.; Onagraceae: Ludwigia peruviana (L.) H. Hara.; Passifloraceae: Turnera subulata Sm.; Phyllanthaceae: Phyllanthus debilis Klein ex Willd.; Poaceae: Bothriochloa pertusa (L.) A. Camus.; Primulaceae: Samolus valerandi L.; Rosaceae: Fragaria sp.; Rubiaceae: Richardia grandiflora (Cham. \& Schltdl.) Steud., Spermacoce verticillata L.; Sapindaceae: Filicium decipiens (Wight \& Arn.) Thwaites; Solanaceae: Capsicum sp. cv. 'Topito', Solanum torvum Sw.; Vitaceae: Ampelopsis arborea (L.) Koehne, Vitis rotundifolia Michx. (EsQUIVEL-Ríos, 2015; EtienNe \& MATIle-Ferrero, 2008; Hempel, 1912; Hodges et al., 2008; KONDO et al., 2016).

REMARKS - Outside South America, C. genistae has been reported from Antigua and Barbuda (MiLler et al., 2014), Bahamas (Hodges, 2006), Barbados (CiOMPERlik 2010; Hodges, 2006), Curacao (VAn BuUrt \& Debrot, 2011), Dominica (Miller et al., 2014), Grenada (Evans \& Dooley, 2013; Miller et al., 2014), Guadeloupe (ETIENNE \& MATILEFERrERo, 2008), Haiti (PESTNET, 2016), Jamaica (Miller et al., 2014), Mexico (Miller et al., 2014), Panama (Esquivel-Ríos, 2015), Puerto Rico (CIOMPERlik, 2010; Evans \& Dooley, 2013; Miller et al., 2014), St. Kitts and Nevis (Miller et al., 2014), Saint Lucia (MalumPhy, 2014), Trinidad and Tobago (Evans \& Dooley, 2013; Miller et al., 2014), USA (Hodges et al., 2008), U.S. Virgin Islands (Evans \& DoOley, 2013; Miller et al., 2014), and West Africa (Evans \& DoOLEy, 2013).

\section{Crypticerya luederwaldti (Hempel)} Icerya luederwaldti Hempel, 1918: 197.

Distribution In SOUth AMERICA - Brazil (HeMPEL, 1918). Pest Status - Unknown.

NATURAL ENEMIES - Not reported.

Host Plants - Hempel (1918) lists the host as a salt- loving plant, with the insects living on the underside of the leaves near the central nerves (main veins).

REMARKS - Only known from Brazil. UNRUH \& GULLAN (2008b) could not examine specimens of $C$. luederwaldti for their identification guide to species of Iceryini.

\section{Crypticerya minima (Morrison)}

Icerya minima Morrison, 1919: 64.

Distribution In South America - Argentina (Lizer Y Trelles, 1938; Morrison, 1919).

Pest Status - Unknown.

Natural EnEmies - Not reported.

Host Plants - Amaranthaceae: Suaeda divaricata Moq. (Lizer Y TReLles, 1938; Morrison, 1919).

REMARKS - Only known from Argentina.

Crypticerya montserratensis (Riley \& Howard) Icerya montserratensis Riley \& Howard, 1890: 99.

Distribution In South America - Colombia (FigueroAPotes, 1946), Ecuador (BARtlett, 1978), Venezuela (GORDON, 1972).

Pest STATUs - Known as a major pest of citrus in Ecuador and a minor pest in Puerto Rico (BARTLETT, 1978).

Natural Enemies - Coleoptera: Coccinellidae: Anovia punica Gordon (Gordon, 1972), Rodolia cardinalis (Mulsant) (BARTLETT, 1978). Diptera: Cryptochaetidae: Cryptochaetum iceryae (Williston) (BARTLETT, 1978). Phoridae: Syneura cocciphila (Coquillett) (BARTLETT, 1978). Hymenoptera: Encyrtidae: Brethesiella sp., Cheiloneurus pulvinariae Dozier, Homosemion bennetti Annecke, Iceromyia flavifrons Noyes (NOYES, 2004).

Host Plants - Arecaceae: Cocos nucifera L.; Calophyllaceae: Calophyllum calaba L., Mammea americana L.; Casuarinaceae: Casuarina equisetifolia L.; Fabaceae: Albizia saman (Jacq.) F. Muell., Inga fagifolia G. Don, I. vera Willd., Prosopis sp.; Lauraceae: Persea americana Mill.; Lythraceae: Lawsonia inermis L.; Malpighiaceae: Byrsonima crassifolia (L.) Kunth.; Moraceae: Ficus microcarpa L. f.; Musaceae: Musa sp.; Myrtaceae: Psidium guajava L., Psidium sp.; Rutaceae: Citrus aurantium L., C. maxima (Burm.) Merr., Citrus sp.; Sapotaceae: Chrysophyllum sp. (FIGUEROA-Potes, 1946; Foldi, 1995; Martorell, 1976; Riley \& Howard, 1890a, b; TOWNSEND \& COCKERELL, 1898).

REMARKS - Outside of South America, C. montserratensis is known from Grenada (VAYSSIÈRE, 1926), Haiti (PEREZGelabert, 2008), Mexico (Foldi, 1995, Cockerell, 1899), Montserrat (RILEY \& HowARD, 1890), Panama (CoCKerell, 1899), and Puerto Rico (MARTORELL, 1976).

Crypticerya multicicatrices Kondo \& Unruh Crypticerya multicicatrices Kondo \& Unruh, 2009: 95.

Distribution In South AMERICA - Colombia (Kondo \& UNRUH, 2009).

Pest Status - Crypticerya multicicatrices is present throughout the year, with infestations being more severe in times of drought (KonDO et al., 2012a). It has been reported associated with stunted growth on soursop, Annona muricata L. (Kondo, 2008), and defoliation and death of the host in severe attacks (KONDO et al., 2012a). The species produces honeydew that promotes the growth of fungi that cause sooty mold, resulting in cosmetic damage to the commercial parts of the plant and reducing the quality of the product. Outbreaks of C. multicicatrices occurred on San Andres Island (Colombia) between 2010 and 2013, until the coccinellid beetle, Anovia sp. successfully controlled this 
insect pest (KONDO et al., 2014). This insect predator was later identified as Anovia punica Gordon (GonZÁlez \& KondO, 2014). In mainland Colombia, outbreaks of $C$. multicicatrices commonly occur in the city of Cali, affecting urban trees such as Caesalpinia pluviosa var. peltophoroides, Pithecellobium dulce and P. saman (Fabaceae) and mango Mangifera indica (Anacardiaceae) (PINCHAO et al., 2015).

NATURAL ENEMIES - Fungi: Eurotiales: Trichocomaceae: Isaria sp. (Kondo et al., 2012a, SILvA-GómEZ et al., 2013). Coleoptera: Coccinellidae: Anovia punica Gordon (GonzÁlez \& Kondo, 2014), Delphastus quinculus Gordon, Diomus seminulus (Mulsant) (GonzÁlez et al., 2012), Rodolia cardinalis (Mulsant) (PINCHAO et al., 2015). Diptera: Phoridae: Syneura cocciphila (Coquillet) (GAIMARI et al., 2012). Hymenoptera: Encyrtidae: Brethesiella cf. abnormicornis (Girault) (MONTEALEGRE et al., 2016), Cheiloneurus sp. (an encyrtid hyperparasitoid). Neuroptera: Chrysopidae: Chrysoperla sp., Ceraeochrysa sp. (Kondo et al., 2014).

Host Plants - Acanthaceae: Avicennia germinans (L.) L., Dicliptera assurgens (L.) Juss., Graptophyllum pictum (L.) Griff., Pseuderanthemum reticulatum (Hort. ex Hook. fil.) Radlk.; Amaranthaceae: Achyranthes sp., Spinacia oleracea L.; Amaryllidaceae: Allium cepa L., A. fistulosum L., Hymenocallis caribaea (L.) Herb.; Anacardiaceae: Mangifera indica L., Spondias mombin L., S. purpurea L.; Annonaceae: Annona cherimola Mill., A. muricata L., Annona squamosa L.; Apocynaceae: Catharanthus roseus (L.) G. Don., Nerium oleander L., Plumeria sp.; Araceae: Adonidia merrillii (Becc.) Becc., Alocasia macrorrhizos (L.) G. Don, Anthurium cubense Engl., Epipremnum aureum (Linden \& André) G.S. Bunting; Araliaceae: Polyscias sp., Schefflera sp.; Arecaceae: Attalea butyracea (Mutis ex L. f.) Wess. Boer, Caryota sp., Cocos nucifera L., C. nucifera L. "Malayan dwarf", Dypsis lutescens $(\mathrm{H}$. Wendl.) Beentje \& J. Dransf., Phoenix roebelenii O'Brien, Pritchardia pacifica Seem. \& H. Wendl., Veitchia sp.; Asparagaceae: Cordyline terminalis (L.) Kunth., Cordyline sp., Dracaena sp., Sansevieria fasciata Cornu ex Gérôme \& Labroy; Asteraceae: Emilia sonchifolia (L.) DC.; Berberidaceae: Nandina domestica Thunb.; Bignoniaceae: Crescentia cujete L., Spathodea campanulata P. Beauv., Tecoma stans (L.) Juss. ex Kunth; Bixaceae: Bixa orellana L.; Boraginaceae: Cordia sebestena L.; Calophyllaceae: Mammea americana L.; Capparaceae: Quadrella odoratissima (Jacq.) Hutch.; Caricaceae: Carica papaya L.; Casuarinaceae: Casuarina equisetifolia L.; Chrysobalanaceae: Licania tomentosa (Benth.) Fritsch; Clusiaceae: Rheedia madruno (Kunth) Planch. \& Triana; Combretaceae: Conocarpus erectus L., Laguncularia racemosa (L.) C.F. Gaertn.; Convolvulaceae: Ipomoea batatas (L.) Lam.; Crassulaceae: Kalanchoe pinnata (Lam.) Pers.; Cucurbitaceae: Cucumis sativus L., Luffa cylindrica (L.) M. Roem.; Cyperaceae: Cyperus ligularis L.; Euphorbiaceae: Acalypha wilkesiana Müll. Arg., Codiaeum variegatum (L.) Rumph. ex A. Juss., Euphorbia aphylla Brouss. ex Willd., Euphorbia hirta L., E. pulcherrima Willd. ex Klotzsch, Jatropha gossypiifolia L., J. integerrima Jacq., Manihot esculenta Crantz; Fabaceae: Albizia lebbeck (L.) Benth., Arachis pintoi Krapov. \& W.C. Greg., Bauhinia monandra Kurz, Caesalpinia peltophoroides Benth., Cajanus cajan (L.) Huth, Calliandra pittieri Standl., Calliandra sp., Cassia fistula L., C. grandis L. f., Delonix regia (Bojer ex Hook.) Raf., Erythrina variegata L., Erythrina sp., Flemingia strobilifera (L.) R. Br., Leucaena leucocephala (Lam.) de Wit, Gliricidia sepium (Jacq.) Kunth ex Walp., Phaseolus vulgaris L., Phaseolus sp., Pithecellobium dulce (Roxb.) Benth., Tamarindus indica L.; Lamiaceae: Ocimum sanctum L., Plectranthus unguentarius Codd, Salvia sp., Tectona grandis L. f.; Lauraceae: Persea americana L.; Musaceae: Musa $\times$ paradisiaca L., Musa x sapientum L.; Lythraceae: Lagerstroemia indica L., Punica granatum L.; Malvaceae: Abelmoschus esculentus (L.) Moench, Ceiba pentandra (L.) Gaertn., Gossypium barbadense L., Guazuma ulmifolia Lam., Hibiscus rosa-sinensis L., Hibiscus sabdariffa L., Hibiscus sp., Malvaviscus arboreus, Thespesia populnea (L.) Sol. ex Corrêa; Moraceae: Artocarpus altilis (Parkinson) Fosberg, Artocarpus heterophyllus Lam., Ficus benjamina L., F. elastica Roxb. ex Hornem., F. lyrata Warb., F. microcarpa L. f., Ficus sp.; Myrtaceae: Pimenta dioica (L.) Merr., Psidium guajava L., Psidium sp., Syzygium jambos (L.) Alston, Syzygium samarangense (Blume) Merr. \& L. M. Perry; Nyctaginaceae: Bougainvillea sp.; Orchidaceae: Brassavola nodosa (L.) Lindl.; Oxalidaceae: Averrhoa carambola L.; Passifloraceae: Passiflora edulis fo. flavicarpa O. Deg.; Phyllanthaceae: Phyllanthus acidus (L.) Skeels; Poaceae: Poa sp., Zea mays L.; Polygonaceae: Coccoloba uvifera (L.); Rubiaceae: Alibertia edulis (Rich.) A. Rich. ex DC., Ixora coccinea L., Morinda citrifolia L.; Rutaceae: Citrus $\times$ aurantiifolia (Christm.) Swingle, C. aurantium L., C. grandis (L.) Osbeck, C. latifolia Tanaka, C. $\times$ limon (L.) Osbeck, C. reticulata Blanco, C. sinensis (L.) Osbeck, Swinglea glutinosa (Blanco) Merr.; Sapindaceae: Melicoccus bijugatus Jacq.; Sapotaceae: Chrysophyllum cainito L., Manilkara zapota (L.) P. Royen; Smilacaceae: Smilax spinosa Mill.; Solanaceae: Capsicum chinense Jacq. (Habanero pepper), Capsicum sp., Cestrum nocturnum L., Solanum lycopersicum Lam., S. melongena L.; Strelitziaceae: Ravenala madagascariensis Sonn.; Urticaceae: Cecropia peltata L., C. schreberiana Miq.; Zingiberaceae: Alpinia purpurata (Vieill.) K. Schum. (Kondo et al., 2012a; SILVA-GomeZ et al., 2013).

REMARKS - Only known from mainland Colombia and San Andres and Providence Islands (Colombian territory). In life, the species resembles $C$. brasiliensis and $C$. zeteki, which are also known to occur in Colombia (see Remarks section under C. brasiliensis).

Crypticerya palmeri (Riley \& Howard) Icerya palmeri Riley \& Howard, 1890: 103.

Distribution in South America - Argentina (Lizer Y Trelles, 1938), Chile (BARTLett, 1978; Lizer Y Trelles, 1938, VAYSSIÈRE, 1926).

Pest STATUs - Crypticerya palmeri has been reported as a serious pest of alfalfa, grapevines and ornamental plants in Chile (BARTLETT, 1978).

NAtURAl EnEmies - Coleoptera: Coccinellidae: Rodolia cardinalis (Mulsant) (BARTLETT, 1978). Hymenoptera: Encyrtidae: Brethesiella flava Timberlake (TIMBERLAKE, 1926).

Host Plants - Fabaceae: Coursetia sp. (Foldi, 1995; TOWNSEND \& COCKERELL, 1898), Medicago sp. (BARTLETT, 1978). Poaceae: undetermined species (LIZER Y TRELLES, 1938). Rosaceae: Acaena argentea Ruiz \& Pav. (LIZER Y TRELlES, 1938). Vitaceae: Vitis vinifera L. (BARTLETT, 1978; RILEY \& HOWARD, 1890).

REMARKS - The species was originally described from specimens collected in Mexico, on grapevines (RILEY \& HowARD, 1890). Later, the species was reported from Argentina and Chile (see distribution above), but whether the South American specimens are conspecific with the 
Mexican species needs to be verified. In their identification guide to species of Iceryini, UNRUH \& GULLAN (2008b) examined only specimens from Mexico (the type locality), thus, currently we do not know whether the South American species are conspecific, and these may be an undescribed species or a different named species.

Crypticerya subandina (Leonardi)

Icerya subandina Leonardi, 1911: 238 .

Distribution In SOUth AMERICA - Argentina (LeONARDI, 1911; LizER Y TRELLES, 1917, 1938).

Pest Status - Unknown.

NATURAL ENEMIES - Not reported.

Host Plants - Fabaceae: Anadenanthera colubrine (Vell.) Brenan (Lizer Y Trelles, 1938); Zygophyllaceae: Bulnesia retama (Gillies ex Hook. \& Arn.) Griseb. (LEONARDI, 1911; LizER Y TRELLES, 1938).

REMARKS - Only known from Argentina.

Crypticerya zeteki (Cockerell) Icerya zeteki Cockerell, 1914: 148.

Distribution In SOUth AMERICA - Colombia (Kondo et al., 2016).

Pest Status - In Colombia, it is listed as a pest of coconut, oil palm and roses by POSADA (1989).

NATURAL EnEMIES - Not reported.

Host Plants - Arecaceae: Dypsis lutescens (H. Wendl.) Beentje \& J. Dransf., Cocos nucifera L., Elaeis guineensis Jacq.; Fabaceae: Trifolium repens L., Arachis pintoi Krapov. \& W.C. Greg.; Rosaceae: Rosa sp. (Kondo et al., 2016; POSADA, 1989).

REMARKS - The species was originally recorded from Panama (Canal Zone) on an undetermined plant (COCKERELL, 1914). The arrangement of waxy secretions in live specimens of $C$. brasiliensis, $C$. multicicatrices and $C$. zeteki is superficially similar, all sharing a long caudal waxy tuft and a shorter cephalic tuft (for more details see KONDO et al., 2016). A discussion of the similarities of C. brasiliensis, C. montserratensis, C. multicicatrices (latter referred to as an undescribed Colombian species) and $C$. zeteki can be found in UNRUH \& GULLAN (2008b).

Icerya chilensis Hempel

Icerya chilensis, Hempel, 1920, 333.

Distribution in South America - Chile (Hempel, 1920).

Pest Status - Unknown.

NATURAL ENEMIES - Not reported.

Host Plants - Collected on the leaves of an undetermined wild tree (HEMPEL, 1920).

REMARKS - Only known from Chile. UnRUH \& GULLAN (2008b) regarded this species as incertae sedis. The natural occurrence of an Icerya species in South America is doubtful. The genus Icerya is native to Indomalaya and Australasia, not South America. In the original description, HEMPEL, (1920) wrote that once the male of this species was discovered, that the species might be put into a different genus.

Icerya insulans Hempel

Icerya insulans Hempel, 1923: 510.

Distribution in South America - Brazil (Hempel, 1923).

Pest Status - Unknown.

NATURAL EnEMIES - Not reported.

Host PlanTs - Asteraceae: Undetermined species; Mela- stomataceae: Tibouchina clavata (Pers.) Wurdack (HEMPEL, 1923).

REMARKS - Only known from Brazil. UNRUH \& GULLAN (2008b) regarded this species as incertae sedis. The natural occurrence of an Icerya species in South America is doubtful as the genus Icerya is native to Indomalaya and Australasia, not South America. In the original description, HEMPEL (1923) writes that this species belongs to the same group as Icerya taunayi Hempel, which is now Laurencella taunayi (Hempel), and thus it is likely that I. insulans may also be a species of Laurencella.

\section{Icerya paulista Hempel \\ Icerya paulista Hempel, 1920: 336.}

Distribution in South America - Brazil (Hempel, 1920).

Pest Status - Unknown.

NATURAL ENEMIES - Not reported.

Host Plants - Poaceae: On the thorns of a plant locally known as "taquarucu", Guadua sp. (Hempel, 1920).

REMARKS - Only known from Brazil. UNRUH \& GULLAN (2008b) regarded this species as incertae sedis. The natural occurrence of an Icerya species in South America is doubtful as the genus Icerya is native to Indomalaya and Australasia, not South America.

Icerya purchasi Maskell

Icerya purchasi Maskell, 1879: 221.

Distribution in South America - Argentina (CABI, 1971; Lizer Y Trelles, 1938; Granara de WillinK \& ClAPS, 2003), Bolivia (CABI, 1971), Brazil (CABI, 1971; Culik et al., 2007; Hempel, 1918, 1920), Chile (CABI, 1971; GonzÁlez, 1989; RIPA et al., 2008), Colombia (CABI, 1971; Figueroa-Potes, 1946; Kondo, 2001; KoNDO et al., 2012b), Ecuador (CABI, 1971; Galapagos island: CAUSTON et al., 2004; LINCANGO et al., 2010), French Guiana (REMILLET, 1988), Paraguay (CABI, 1971), Peru (BEDERSKI, 1969; CABI, 1971; LINDINGER, 1942), Uruguay (CABI, 1971, Lizer Y Trelles, 1938), Venezuela (CABI, 1971).

Pest STATUS - Icerya purchasi is a well-known pest of citrus and many other plants (CALTAGIRONE, 1981; Watson \& MALUMPHY, 2004). In recent years it was reported as a serious pest in the Galapagos Islands, where it was successfully controlled by the vedalia beetle, Rodolia cardinalis (Mulsant) (CAUSTON et al., 2004). In Colombia, I. purchasi is rather rare and generally not considered a pest of citrus (Kondo et al., 2012b). In Brazil, I. purchasi has been found on Citrus spp. in the State of São Paulo and various ornamental plants (ALMEIDA et al., 2015).

Natural Enemies - Hemiptera: Heteroptera: Anthocoridae: Cardiastethus nazarenus Reuter (AwADALLAH et al., 1976). Coleoptera: Coccinellidae: Anovia punica Gordon (Gordon, 1972), Chilocorus bipustulatus (L.) (ARGYRIOU et al., 1976), Cryptolaemus montrouzieri Mulsant (JASHENKO, 1999; Puttarudriah \& Channabasavanna, 1957), Hippodamia sp. (JASHENKO, 1999), Rodolia cardinalis (Mulsant) (Brain \& Kelly, 1917; Gullan \& Cranston, 2014; ZIMMERMAN, 1948), Rodolia pumila Weise (ChIU et al., 1985), Scymnus sp. (Fullaway, 1913). Diptera: Cryptochaetidae: Cryptochaetum iceryae (Williston) (Gullan \& Cranston, 2014; Mendel \& BlumberG, 1991; Quezada \& Debach, 1973; Denmark, 1964), Phoridae: Syneura cocciphila (Coquillet) (Autuori, 1928). Hymenoptera: Aphelinidae: Ablerus molestus Blanchard, Coccophagus lycimnia Walker, Coccophagus scutellaris 
Dalman (NoyEs, 2004, 2016). Encyrtidae: Brethesiella abnormicornis Girault, B. latifrons Timberlake, Cheiloneurus pulvinariae Dozier, Homalotylus eytelweinii Ratzeburg, H. flaminius Dalman, Homosemion bennetti Annecke, Isodromus iceryae Howard, Lamennaisia ambigua Nees, Tetracnemoidea brevicornis Girault (NoYes, 2004, 2016). Eriaporidae: Euryischia sp., Euryischia lestophoni Riley (NoYES, 2016). Eulophidae: Entedon coquillettii Riley (NOYES, 2004, 2016). Mymaridae: Alaptus iceryae Riley (Noyes, 2004, 2016). Pteromalidae: Austroterobia sp., Ophelosia crawfordi Riley, O. hypatia Girault, Oricoruna arcotensis Mani \& Kurian, Parasaphodes townsendi Ashmead, Parasaphodes sp. (Noyes, 2004, 2016). Lepidoptera: Pyralidae: Laetilia coccidivora (Comstock) (JASHENKo, 1999). Thysanoptera: Phlaeothripidae: Megalothrips sp. (JASHENKO, 1999). Neuroptera: Chrysopidae: Chrysopa pallens Rambur (JASHENKO, 1999), C. sapporensis Okamoto (JASHENKO, 1999), Pseudomallada ventralis (Curtis) (Jashenko, 1999), Semachrysa matsumurae (Okamoto) (JASHENKO, 1999; KUWANA, 1922).

Host PLANTS - This species is highly polyphagous. It has been recorded on more than 230 species of plants distributed in 162 genera in 66 plant families (GARCíA MORALES et al., 2016).

REMARKS - Of Australian origin, I. purchasi has now spread worldwide with infested plant material. In Colombia, the cottony cushion scale Icerya sp. (possibly I. purchasi Maskell) is considered as one of the first urban pests (KondO, 2015). According to VAlEnZuela (1993), on the occasion of the celebration of the IX Pan American Conference held in Bogota in 1948, a number of acacia trees were introduced to Colombia in order to beautify the streets of the capital city; however, the imported plants came infested with an insect pest, Icerya sp., for which populations increased to such an extent that it did not take long before the citizens started calling it the "white plague". Fortunately, a successful classical biological control program was implemented through the importation of the lady beetle $R$. cardinalis (Mulsant) (VALENZuela, 1993). Several subspecies of $I$. purchasi can be found in the literature (i.e., Icerya purchasi citriperda Hempel, I. purchasi crawii Cockerell and I. purchasi maskelli Cockerell), however, there is no evidence to recognize subspecies of $I$. purchasi and thus the name I. purchasi citriperda Hempel and other trinomens should not be used (GULLAN \& KONDO, 2011).

\section{Icerya schrottkyi Hempel}

Icerya schrottkyi Hempel, 1900: 373.

Distribution in South America - Argentina (Lizer Y Trelles, 1936, 1938), Brazil (Foldi \& Soria, 1989; HeMPEL, 1900).

Pest Status - Considered a pest of grapevines in Brazil (FOLDI \& SORIA, 1989).

NATURAL ENEMIES - Not reported.

Host Plants - Euphorbiaceae: Sebastiania commersoniana (Baill.) L.B. Sm. \& Downs (Lizer Y Trelles, 1938); Vitaceae: Vitis vinifera L. (FoldI \& SORIA, 1989).

REMARKS - UNRUH \& GULLAN (2008b) included this species in the "Icerya aegyptiaca group" which includes two species, i.e., I. aegyptiaca and I. schrottkyi. The adult females of both species have pores on the derm that resemble the vulvar pores, but I. schrottkyi possesses unique hexalocular pores on the ventral surface that are absent from all other iceryine species. The two species can be separated easily by the number of cicatrices on the venter of the adult female (one in I. aegyptiaca, three in I. schrottkyi) (UNRUH \& GULLAN, 2008b). The native distribution of this species may not be Brazil.

Icerya seychellarum (Westwood)

Dorthesia seychellarum Westwood, 1855: 836.

Distribution in South America - Colombia (FigueroAPotes, 1946), French Guiana (WiLliams \& WATSON, 1990).

Pest StATUs - This species has been reported from Fiji as a pest of guava and citrus (Citrus spp.) (LEVER, 1946) and Rosa sp. (HINCKLEY, 1965). It has also been reported killing citrus trees in Vanautu (Williams \& Butcher, 1987). Icerya seychellarum may produce copious amounts of honeydew that serve as a medium for sooty molds that grow on the leaves, reducing plant respiration and photosynthesis (COHIC, 1955).

NAtURAL EnEmies - Coleoptera: Coccinellidae: Rodolia cardinalis (Mulsant) (BARTLETT, 1978), R. limbata (Blackburn) (BARTLETT, 1978); R. pumila Weise (Beardsley, 1955). Diptera: Cryptochaetidae: Cryptochaetum iceryae (Williston) (BARTLETT, 1978); Hymenoptera: Encyrtidae: Zaplatycerus tachikawai Subba Rao (NoYES, 2004, 2016). Eriaporidae: Euryischia indica Mani \& Kurian (Noyes, 2004, 2016). Eulophidae: Aprostocetus pauliani Risbec (Noyes, 2004, 2016). Pteromalidae: Ophelosia crawfordi Riley, Parasaphodes townsendi Ashmead (Noyes, 2004, 2016).

Host Plants - Icerya seychellarum is a highly polyphagous species, recorded from 166 plants distributed in 126 genera in 57 families (GARCÍA MorALES et al., 2016).

REMARKS - In South America, it has been reported only from Colombia, where is considered to be an introduction. This is a widespread species in the Pacific region especially in the tropical South Pacific area, throughout Africa and southern Asia on many plant species (WILLIAMS \& WATSON, 1990). The species was redescribed and illustrated by UNRUH \& GULLAN (2008a).

\section{ACKNOWLEDGEMENTS}

Many thanks to Dr. Cory M. Unruh for providing literature on iceryine scale insects. The first author thanks the Colombian Corporation for Agricultural Research (Corpoica) for funding his trip to Catania, Italy, in order to participate in the XIV International Symposium on Scale Insect Studies - ISSIS. Thanks also to anonymous reviewers for useful comments that helped improve the manuscript.

\section{REFERENCES}

Almeida L.F.V., Peronti A.L.B.G., Martinelli N.M,. 2015 - Estudo diagnóstico e taxonômico das cochonilhas (Hemiptera: Coccoidea) associadas às plantas citrícas no estado de São Paulo. In: III Congresso Brasileiro de Fitossanidade, 2015, Águas de Lindóia. Anais do III Congresso Brasileiro de Fitossanidade, CD-Rom.

Argyriou L.C., StaVraKi H.G., Mourikis P.A., 1976 - A List of Recorded Entomophagous Insects of Greece. Benaki Phytopathological Institute, Athens. 73 pp.

Autuori M., 1928 - Syneura infraposita Borgm.-Schmitz (Diptera, Phoridae): um novo parasita da Icerya purchasi Mask. - Archivos do Instituto Biologico, São Paulo, 1: 193-200.

Awadallah K.T., Tawfik M.F.S., Swailem S.M., El 
Maghraby M.M.A., 1976 - The effect of feeding on various preys on the nymphal stage and oviposition of Cardiastethus nazarenus Reuter (Hemiptera-Heteroptera: Anthocoridae). - Bulletin of the Entomological Society of Egypt, 1980 (60): 251-255.

BARTLETT B.R., 1978 - Margarodidae. Introduced Parasites and Predators of Arthropod Pests and Weeds: a World Review. - Agricultural Research Service, United States Department of Agriculture Washington, D.C., 545 pp.

BEARDSLEY J.W., 1955 - Fluted scales and their biological control in United States administered Micronesia. Proceedings of the Hawaiian Entomological Society, 15: 391-399.

BEDERSKI K.A., 1969 - Insect population surveys and oil rotenone sprays: their use in Peruvian citrus orchards. Proceedings First International Citrus Symposium, Vol. 2, University of California Riverside, pp. 933-940.

BRAIN C.K., Kelly A.E., 1917 - The status of introduced coccids in South Africa in 1917. - Bulletin of Entomological Research, 8: 181-185.

CABI, 1971 - Icerya purchasi (Mask.). Distribution Maps of Pests, Series A, Agricultural Map No. 51 (rev.): 3 pp.

CALTAGirone L.E., 1981 - Landmark examples in classical biological control. - Annual Review of Entomology, 26: 213-232.

Causton C.E., Lincango M.P., Poulsom T.G.A., 2004 Feeding range studies of Rodolia cardinalis (Mulsant), a candidate biological control agent of Icerya purchasi Maskell in the Galapágos Islands. - Biological Control, 29: 315-325.

Chiu S.-C., Lo K.-C., Ching-Chin C., Chen C.-C., Chen C.-F., 1985 - Biological Control of Citrus Pests in Taiwan. - Taiwan Agricultural Research Institute, Taiwan. Special publication No. 19: 1-8.

Ciomperlik M., 2010 - Crypticerya genistae scale, an invasive pest in Puerto Rico. In: CPHST Biological Control Unit 2010. - Annual Report, U. S. Department of Agriculture, pp. 33-34. Available at: http://www.aphis. usda.gov/plant_health/cphst/downloads/2010BiologicalC ontrolUnitAnnualReport.pdf (Accessed on August 12, 2016).

Cockerell T.D.A., 1899 - Rhynchota, Hemiptera Homoptera. [Aleurodidae and Coccidae]. - Biologia Centrali-Americana, 2: 1-37.

Cockerell T.D.A., 1914 - A new cotton scale from Panama. - Journal of Economic Entomology, 7: 148.

CoHIC F., 1955 - Rapport d'une mission aux etablissements français de l'ocëanie. Fascicule III. Enquête sur les parasites animaux des cultures. Institut francais d'Oceanie, Nouméa (Nouvelle-Calédonie), 68 pp.

Culik M.P., Martins D.S., Ventura J.A., Peronti A.L.B.G., Gullan P.J., Kondo T., 2007 - Coccidae, Pseudococcidae, Ortheziidae, and Monophlebidae (Hemiptera: Coccoidea) of Espirito Santo, Brazil. - Biota Neotropica, 7 (3): 1-5.

Denmark H.A., 1964 - Florida Department of Agriculture's position on parasites and predators introduced into Florida for biological control. - Hyacinth Control Journal, 3: 16-19.

EsQuivel-Ríos E.A., 2015 - Crypticerya genistae Hempel (Hemiptera: Margarodidae) sobre Mani forrajero Arachis pintoi (Fabaceae) en Panamá Oeste. Agrociencia Panamensis. 1 p. Available at: http://agrocienciapanama.blogspot.com.co/2015/06/crypticerya-genistaehempel-hemiptera.html

Etienne J., Matile-Ferrero D., 2008 - Crypticerya genistae (Hempel), nouveau danger en Guadeloupe
(Hemiptera, Coccoidea, Monophlebidae). - Bulletin de la Société Entomologique d'Egypte, 113 (4): 517-520.

Evans G.A., Dooley J.W., 2013 - Potential invasive species of scale insects for the USA and Caribbean Basin. In: Peña JE. Ed. Potential invasive pests of agricultural crops. CABI, Wallingford, pp. 320-341.

Figueroa-Potes A., 1946 - Catalogación inicial de las cochinillas del Valle del Cauca (Homoptera - Coccoidea). - Revista Facultad de Agronomía, Montevideo Universidad, 6: 196-220.

FoldI I., 1995 - Margarodidae du Mexique (Hemiptera: Coccoidea). - Annales de la Société Entomologique de France, 31: 165-178.

FOLDI I., 2009 - Archaeococcoid scale insects (Hemiptera: Coccoidea) from the tropical high mountains of the Andean Cordillera, South America. - Zootaxa, 2300: 1-38.

FOLDI I., SORIA, S.J., 1989 - Les cochenilles nuisibles a la vigne en Amerique du Sud (Homoptera: Coccoidea). Annales de la Société Entomologique de France (N.S), 25 (4): 411-430.

FullawaY D.T., 1913 - Entomological notes. - Annual Report. Guam Agricultural Experiment Station for 1911: 26-35.

Gagné R.J., EtiEnNe J., 2009 - Note on the Cecidomyiidae from Guadeloupe (West Indies) with description of a new species of Paracalmonia (Diptera). - Bulletin de la Société Entomologique de France, 114(3): 337-350.

Gaimari S.D., Quintero E.M., Kondo T., 2012 - First report of Syneura cocciphila (Coquillett, 1895) (Diptera: Phoridae), as a predator of the fluted scale Crypticerya multicicatrices Kondo \& Unruh, 2009 (Hemiptera: Monophlebidae). - Boletín del Museo de Entomología de la Universidad del Valle, 13(2): 26-28.

García Morales M., Denno B., Miller D.R., Miller G.L., Ben-Dov Y., Hardy N.B., 2016 - ScaleNet: A Literature-based Model of Scale Insect Biology and Systematics. Available at: http://scalenet.info.

GONZÁLEZ R.H., 1989 - Insectos y ácaros de importancia agrícola y cuarentenaria en Chile. Universidad de Chile Santiago, $310 \mathrm{pp}$.

GonZÁLEZ G., KONDO T., 2014 - Geographical distribution and phenotypic variation of Anovia punica Gordon (Coleoptera: Coccinellidae: Noviini), a predatory ladybeetle of fluted scales (Hemiptera: Coccoidea: Monophlebidae). - Insecta Mundi, 0398: 1-6.

GONZÁlEZ G., LÓPEZ R., KONDO T., 2012 - First report of Delphastus quinculus Gordon and Diomus seminulus (Mulsant) (Coleoptera: Coccinellidae) feeding on eggs and first-instar nymphs of Crypticerya multicicatrices Kondo \& Unruh (Hemiptera: Monophlebidae). - Insecta Mundi, 0268: 1-6.

GORDON R.D., 1972 - The tribe Noviini in the new world (Coleoptera: Coccinellidae). - Journal of the Washington Academy of Sciences, 62 (1): 23-31.

Granara De Willink M.C., Claps L.E., 2003 Cochinillas (Hemiptera: Coccoidea) presentes en plantas ornamentales de la Argentina. - Neotropical Entomology, 32 (4): 625-637.

Gullan P.J., Cranston P.S., 2014 - The Insects: An Outline of Entomology, 5th Edition. Wiley-Blackwell, West Sussex, UK, 595 pp.

Gullan P., Kondo T., 2011 - Nomenclator entomologicus. No. 79. [In Portuguese]. Informativo da Sociedade Entomológica do Brasil. February, 36(1): 3.

Hempel A., 1900 - As coccidas Brasileiras. - Revista do Museu Paulista, São Paulo 4: 365-537.

Hempel A., 1912 - Catalogos da fauna Brasileira editados pello Museu Paulista, São Paulo - Brasil, 77 pp. 
Hempel A., 1918 - Descripção de sete novas espécies de coccidas. - Revista do Museu Paulista, São Paulo, 10: 193-208.

Hempel A., 1920 - Descripçoes de coccidas novas e pouco conhecidas. - Revista do Museu Paulista, São Paulo, 12: 329-377.

Hempel A., 1923 - Hemiptera (Homoptera) familia Coccidae - Sub-familia Monophlebinae. - Revista do Museu Paulista, São Paulo, 13: 510-512.

Hempel A., 1932 - Descripção de vinte a duas espécies novas de coccideos (Hemiptera - Homoptera). - Revista de Entomologia, 2: 310-339.

HiNCKLEY A.D., 1965 - Trophic records of some insects, mites, and ticks in Fiji. - Bulletin, Department of Agriculture, Fiji, 45: 1-116.

Hodges G., 2006 - Icerya genistae (Hemiptera: Margarodidae) A new exotic scale insect for Florida. Available at: http://www.doacs.state.fl.us/pi/enpp/ento/i. genistae.html.

Hodges G.S., Hodges A.C., UnRuh C.M., 2008 - A new exotic pest for Florida's natural areas: Crypticerya genistae (Hemiptera: Monophlebidae). - Florida Entomologist, 91 (2): 335-337.

JASHENKO R.V., 1999 - [Natural enemies of margarodids in northern Eurasia.] [Problems of Conservation and Sustainable Use of Animal Biodiversity in Kazakhstan. (Proceedings of an International Scientific Conference.)] Tethys Society Almaty, 198 pp.

Jenkins D.A., Mizell R.F. III, Van Bloem S., Whitmire S., Wiscovitch L., Zaleski C., Goenaga R., 2014 - An analysis of arthropod interceptions by APHIS-PPQ and customs and border protection in Puerto Rico. American Entomologist, 60: 44-55.

Kondo T., 2001 - The scale insects of Colombia (Hemiptera: Coccoidea). Biota Colombiana, 2(1): 31-48.

Kondo T., 2015 - Insectos plaga del árbol urbano con énfasis en los insectos escama (Hemiptera: Coccoidea) en Colombia. 17 pp. Simposio: Entomología Forestal. 41 Congreso de la Sociedad Colombiana de Entomología (SOCOLEN). Julio 29-31, 2015. Universidad Nacional de Colombia, Sede Medellín, Medellín, Colombia, 329 pp.

Kondo T., Gullan P., GonzÁlez G., 2014 - An overview of a fortuitous and efficient biological control of the Colombian fluted scale, Crypticerya multicicatrices Kondo \& Unruh (Hemiptera: Monophlebidae: Iceryini), on San Andres island, Colombia. - Acta Zoologica Bulgarica. Suppl. 6: 87-93.

Kondo T., Gullan P., Portilla A.A.R., 2012 - Report of new invasive scale insects (Hemiptera: Coccoidea), Crypticerya multicicatrices Kondo and Unruh (Monophlebidae) and Maconellicoccus hirsutus (Green) (Pseudococcidae), on the islands of San Andres and Providencia, Colombia. - Insecta Mundi, 0265: 1-17.

Kondo T., Gullan P.J., Peronti A.L.B.G., RamosPortilla A.A., Caballero A., Pretelt N.V., 2016 First records of the iceryine scale insects Crypticerya brasiliensis (Hempel) and Crypticerya genistae (Hempel) (Hemiptera: Monophlebidae) for Colombia. - Insecta Mundi, 0480: 1-9.

Kondo T., Peronti A.L., KozÁr F., Szita E., 2012b Capitulo 7. Los insectos escama asociados a los citricos, con énfasis en Praelongorthezia praelonga (Douglas) (Hemiptera: Coccoidea: Ortheziidae) In: Pássaro Carvalho C.P. Ed. Cítricos: Cultivo, Poscosecha e Industrialización. Serie Lasallista Investigación y Ciencia. Artes y Letras S.A.S., Itagüí, Colombia, pp. 173-189.
Kondo T., UnRUh C.M., 2009 - A new species of Crypticerya Cockerell (Hemiptera: Monophlebidae) from Colombia, with a key to species of the tribe Iceryini found in South America. - Neotropical Entomology, 38 (1): $92-$ 100

KuwanA S.I., 1922 - Studies on Japanese Monophlebinae. Contribution II: The genus Icerya. - Bulletin of Agriculture and Commerce, Imperial Plant Quarantine Station, Yokohama, 2: 1-43.

LEONARDi G., 1911 - Contributo alla conoscenza delle coccinglie della Repúbblica Argentina. - Bollettino del R. Laboratorio di Entomologia Agraria di Portici, 5: $237-$ 284

LEVER R.J.A.W., 1946 - Insect pests in Fiji. - Bulletin Department of Agriculture, Fiji, 16: 41-44.

Lincango P., Hodgson C.J., Causton C., Miller D.R., 2010 - An updated checklist of scale insects (Hemiptera: Coccoidea) of the Galapagos Islands, Ecuador. Galapagos Research, 67: 3-7.

LinDINGER L., 1942 - Coccoidea (Homopt.). - Beiträge zur Fauna Perus Bd., 3: 112-122.

Lizer Y Trelles C.A., 1917 - Description du male de l'”Icerya subandina" Leon. Physis, Buenos Aires, 3: 245247.

Lizer Y TRelles C.A., 1936 - Algunas cochinillas nuevas para la fauna de la República Argentina. - Physis, Buenos Aires, 12: 113-116.

Lizer Y Trelles C.A., 1938 - Cochinillas exóticas introducidas en la República Argentina y daños que causan. - Jornadas Agronómicas y Veterinarias, 1937: 341-362.

Malumphy C., 2014 - An annotated checklist of scale insects (Hemiptera: Coccoidea) of Saint Lucia, Lesser Antilles. - Zootaxa, 3846(1): 69-86.

MARTORELL L.F., 1976 - Annotated food plant catalog of the insects of Puerto Rico. Agricultural Experiment Station, University of Puerto Rico, Department of Entomology, 303 pp.

MASKELl W.M., 1879 - On some Coccidae in New Zealand. Transactions and Proceedings of the New Zealand Institute, 11: 187-228

Mendel Z., Blumberg D., 1991 - Colonization trials with Cryptochetum iceryae and Rodolia iceryae for improved biological control of Icerya purchasi in Israel. Biological Control, 1: 68-74.

Miller D.R., Rung A., Parikh G., 2014 - Crypticerya genistae (Hempel). Fact Sheet. In: Scale Insects, edition 2, a tool for the identification of potential pest scales at U.S.A. ports-of-entry (Hemiptera, Sternorrhyncha, Coccoidea). Available at: http://idtools.org/id/ scales/factsheet.php?name $=7063$.

Montealegre X., Sotelo P.; Kondo T., 2016 Development and reproduction of Brethesiella cf. abnormicornis (Girault) (Hymenoptera: Encyrtidae), a parasitoid of the Colombian fluted scale, Crypticerya multicicatrices Kondo \& Unruh (Hemiptera: Monophlebidae). - Environmental Entomology, 8 pp. doi: 10.1093/ee/nvw004.

MORRISON H., 1919 - A report on collection of Coccidae from Argentina with descriptions of apparently new species (Hom.). Proceedings of the Entomological Society of Washington, 21: 63-91.

Newstead R., 1917 - Observations on scale-insects (Coccidae) - $I V$. Bulletin of Entomological Research, 8: 1-34.

Noyes J.S., 2004 - Encyrtidae of Costa Rica (Hymenoptera: Chalcidoidea), 2. Metaphycus and related genera, parasitoids of scale insects (Coccoidea) and 
whiteflies (Aleyrodidae). - Memoirs of the American Entomological Institute, 73: 1-439.

Noyes J.S., 2016 - Universal Chalcidoidea Database. World Wide Web electronic publication. http://www. nhm.ac.uk/chalcidoids.

Palomar J., Peronti A.L.B.G., Soares S.T., Rosa K.C.C., Martinelli N.M., 2015 -Cochonilhas (Hemiptera: Coccoidea) associados à Psidium guajava no Estado de São Paulo. In: III Congresso Brasileiro de Fitossanidade, 2015. Anais do III Congresso Brasileiro de Fitossanidade. CD-Rom.

Perez-Gelabert D.E., 2008 - Arthropods of Hispaniola (Dominican Republic and Haiti): a checklist and bibliography. - Zootaxa, 1831: 1-530.

Peronti A.L.B.G., Rosa K.C.C., 2006 - Cocóideos (Hemiptera: Coccoidea) associados à Psidium guajava (Myrtaceae) em áreas urbanas. In: 19a Reunião Anual do Instituto Biológico, 2006, São Paulo. Anais da 19a Reunião Anual do Instituto Biológico, v. 68, supplement, p. $735-738$

PESTNET, 2016 - Soybean scale, Crypticerya genistae, Haiti. 1 p. Available at: http://www.pestnet.org/ SummariesofMessages/Crops/Vegetables/Soybean/Soybe anscale,Crypticeryagenistae,Haiti.aspx

Pinchao E.C., Kondo T., GonzÁlez F.G., 2015 - Rodolia cardinalis (Mulsant) (Coleoptera: Coccinellidae), a new predator of Crypticerya multicicatrices Kondo and Unruh (Hemiptera: Monophlebidae). - Insecta Mundi, 0431: 1-7.

Posada L.O., 1989 - Lista de insectos dañinos y otras plagas en Colombia. 4ta ed., Bogotá. Boletín Técnico 43. Instituto Colombiano Agropecuario, $662 \mathrm{pp}$.

Puttarudriah M., Channabasavanna G.P., 1957 - Notes on some predators of mealybugs (Coccidae: Hemiptera). - Mysore Agricultural Journal, 32 (1): 4-19.

Quezada J.R., DeBach P., 1973 - Bioecological studies of the cottony cushion scale, Icerya purchasi Mask. and its natural enemies Rodolia cardinalis Mul. and Cryptochaetum iceryae Will., in southern California. Hilgardia, 41: 631-688.

Remillet M., 1988 - Catalogue des insectes ravageurs des cultures en Guyane Française. Editions de l'ORSTOM; Institut Française de Recherche Scientifique pour le Développement en Coopérat Paris, 235 pp.

RiLey C.V., Howard L.O., 1890a - An Icerya in Florida. Insect Life 2: 333.

RILEY C.V., Howard L.O., 1890b - Some new iceryas. Insect Life, 3: 92-106.

RIPA R., LARRAL P., RoJA S., 2008 - Conchuela acanalada, cotton cushion scale. Icerya purchasi (Maskell). Manejo de plagas en paltos y citricos. Instituto de Investigaciones Agropecuarias, Ministerio de Agricultura, Colección Libros INIA-No. 23. La Cruz, Chile, 399 pp.

Silva-Gomez M., Quiroz-GamboA J.A., YePes F.C., MaYA M.F., Santos A., Hoyos-Carvajal L.M., 2013 Incidence evaluation of Crypticerya multicicatrices and Maconellicoccus hirsutus in Colombian Seaflower Biosphere Reserve. - Agricultural Science, 4(12): 654-665.
Stocks I.C., 2013 - Recent Adventive Scale Insects (Hemiptera: Coccoidea) and Whiteflies (Hemiptera: Aleyrodidae) into Florida and the Caribbean Basin. In: Peña J.E. Ed. Potential Invasive Pests of Agricultural Crops. CAB International, Wallingford, UK, pp. 342-362.

TimBerlaKe P.H., 1926 - Miscellaneous new chalcid-flies of the hymenopterous family Encyrtidae. - Proceedings of the United States National Museum, 69 (3): 1-37.

Townsend C.H.T., Cockerell T.D.A., 1898 - Coccidae collected in Mexico by Messrs. Townsend and Koebele in 1897. - Journal of the Entomological Society of New York, 6: 165-180.

TRJaPITZIN V.A., TRJaPITSYN S.V., 2006 - A new species of Brethesiella (Hymenoptera: Encyrtidae) from California, USA, a parasitoid of Steatococcus tabernicolus (Hemiptera: Margarodidae). - Zootaxa, 1167: 1-16.

UNRUH C.M., GULlan P.J., 2008a - Molecular data reveal convergent reproductive strategies in iceryne scale insects (Hemiptera: Coccoidea: Monophlebidae), allowing the re-interpretation of morphology and a revised generic classification. - Systematic Entomology, 33: 8-50.

UnRUh C.M., Gullan P.J., 2008b - Identification guide to species in the scale insect tribe Iceryini (Coccoidea: Monophlebidae). - Zootaxa, 1803: 1-106.

Valenzuela G., 1993 - Aspectos históricos del control biológico. In: Palacios F. Ed. Control biológico en Colombia: historia, avances y proyecciones. - Universidad Nacional de Colombia, Palmira, Colombia, pp. 1-8.

Van BuUrt G. Debrot A.O., 2011 - Introduced agricultural pests, plant and animal diseases and vectors in the Dutch Caribbean, with an "Alert species" list. Report number C193/11. IMARES Wageningen UR. 35 pp. Available at: http://www.dcbd.nl/sites/www.dcbd. $\mathrm{nl} /$ files/documents/C\%252011\%2520193\%2520diseasvec tor\%2520Dutch\%2520Caribb.pdf.

VAYSSIĖRE P., 1926 - Contribution à l'étude biologique et systématique des Coccidae. - Annales des Epiphyties, 12: 187-382.

WATSON G.W., MALumPhy C.P., 2004 - Icerya purchasi Maskell, cottony cushion scale (Hemiptera: Margarodidae), causing damage to ornamental plants growing outdoors in London. - British Journal of Entomology and Natural History, 17: 105-109.

Westwood J.O., 1855 - The Seychelles Dorthesia. Gardeners' Chronicle and Agricultural Gazette, 51: 836.

Williams D.J., Butcher C.F., 1987 - Scale insects (Hemiptera: Coccoidea) of Vanuatu. - New Zealand Entomologist, 9: 88-99.

Williams D.J., Watson G.W., 1990 - The Scale Insects of the Tropical South Pacific Region. Pt. 3: The Soft Scales (Coccidae) and Other Families. CAB International Wallingford. 267 pp.

ZIMMERMAN E.C., 1948 - Homoptera: Sternorrhyncha. Insects of Hawaii, 5: 1-464. 
